Harold A. McAlister and William I. Harkopf (eds.)

\title{
HIPPARCOS First Results in the Double Star Photometry
}

\author{
F. MIGNARD, J. L. FALIN, \& M. FROESCHLÉ \\ Observatoire de la Côte d'Azur / CERGA, \\ Av. Copernic, F06130 Grasse, France
}

\begin{abstract}
In addition to its astrometric capabilities the HIPPARCOS main detector proves to be a good phototometer. The main features of the photometric reduction applied to double stars are outlined. We show how it is possible to discriminate single stars from multiple stars from the photometric signal. Results presented are based on about 16000 stars recognized as non-single, of which 8000 were not known to be double in the HIPPARCOS Input Catalogue. Magnitudes for multiple systems as a whole are derived with a precision usually better than 0.005 mag while the magnitude of each component is recovered up to magnitude difference of 3 mag, but with less accuracy and precision.
\end{abstract}

\section{INTRODUCTION}

The HIPPARCOS satellite launched by ESA on August 1989, has been primarily thought as a powerful instrument to carry out space astrometry at the milliarcsecond level. However, designed as an instrument working by counting photons entering the telescope, HIPPARCOS has the capability of measuring star brightness as well. Thus any stretch of starlight recorded by HIPPARCOS is analysed in view of extracting the photometric information in addition to the positional information.

This photometry is essentially achieved by counting the number of photons crossing the entrance pupil per unit of time and collected by the detector located behind the main grid. Then these numbers are converted into a magnitude defined by a set of standard stars and a model of the instrument response. Details on this processing are available in Mignard et al. $(1989,1992)$ along with a summary of the results already obtained on single stars. In the following we specialize on aspects relevant to double and multiple stars photometry. However we start with a brief summary of the HIPPARCOS photometric processing, which applies without modification to the multiple star, taken as an unresolved source of light in the sky. Then we proceed to the double and multiple star problem to determine the apparent luminosity of each component.

\section{THE PHOTOMETRIC SIGNAL}

About every $2 s$ the photons counts for each star observed are fitted to a five parameter model (Murray et al. 1989) with two harmonics. The global signal $S(t)$ is,

$$
S(t)=I+B+I M \cos (\omega t+\phi)+I N \cos (2 \omega t+\psi)
$$

where $I$ is the total intensity, $B$ the unmodulated background, $M$ and $N$ the modulation coefficients of the first and second harmonic respectively and $\phi$ and $\psi$ the corresponding phases. For a single star we have typically $M=0.72$ and 
$N=0.25$ although these numbers suffers variation of $\pm 7 \%$ with the star color and field position. Thus the photometric information appears in $I, I M$ and $I N$ and must be extracted from the signal. Once this is done the HIPPARCOS magnitude is computed with the usual definition

$$
H_{p}=-2.5 \log _{10} \frac{I}{I_{0}}
$$

where $I_{0}$ is an arbitrary reference intensity. In the following this reference is chosen such that

$$
H_{p}=0 \equiv I_{0}=7 \times 10^{6} \mathrm{~Hz} \text {. }
$$

However, this scaling is completely arbitrary and just a convenient number put into the software which allows the Poisson photon noise to be readily evaluated. The actual definition lies in the computation of the $H$ magnitude for a well defined set of stars, referred to as the photometric standard stars. (Grenon 1991).

The typical background noise is of the order of $35 \pm 15 \mathrm{~Hz}$, depending mainly on the star galactic latitude and the satellite altitude. Its average value over a full revolution of the satellite about its spin axis $(=2.13 h)$ is well determined, whereas fluctuations about the mean are poorly ascertained. As for the observation time allocation $t$, there are in standard conditions four stars crossing the grid at a time in the two overlapping fields of view. Therefore one can allocate about $4 \mathrm{sec}$ of observation per transit for an average star of magnitude 8 . A larger allocation of $\sim 12 \mathrm{sec}$ is available for fainter stars and a smaller $\sim 1 \mathrm{sec}$ for the brightest ones. The detector can track the path of one star at a time, but a computer controlled device allows to sample light of all programme stars in the field of view during their passage across the field.

Each star transiting on the HIPPARCOS grid results in one determination of the magnitude; for a given star, observations are repeated at different intervals $(20 \mathrm{~m}, 2 \mathrm{~h}, 3$ weeks etc. ...) over the mission duration, the peculiarities of which are tied to the HIPPARCOS unusual scanning law. A careful instrumental calibration performed every ten hours is used to get rid of the sensitivity variations with the image location and of the effects brought about by the aging of the instrument. This in turn permits to combine observations performed at very different times. Altogether an average star will be observed about 150 transits during the HIPPARCOS mission. The precision obtained per transit are shown in Table 1 as a function of the star magnitude.

TABLE 1. Photometric precision for one field transit and after one year of mission

\begin{tabular}{|lllllrrrrrr|}
\hline Magnitude & 4 & 5 & 6 & 7 & 8 & 9 & 10 & 11 & 12 & 13 \\
One transit (0.001 mag) & 2 & 4 & 4 & 7 & 10 & 14 & 17 & 30 & 45 & 70 \\
One year (0.001 mag) & 1 & 1 & 1 & 1.5 & 2 & 3 & 4 & 7 & 12 & 25 \\
\hline
\end{tabular}

The processing of one year of data has shown that the above figures are realistic, and provided systematic errors can be kept at such a low level, one can 
benefit from a tremendous statistical improvement by combining observations of constant stars into a single, better defined, magnitude. For those stars we obtain a precision of 0.001 magnitude for a $6^{\text {th }}$ mag star and 0.004 for a $10^{\text {th }}$ mag star. It is not clear at the moment whether we have come close to the limit as noise not related to photon noise will take over and prevent from improving the determination. However the main interest of having a reliable and stable photometry over the misison duration is the study of variable stars in particular when the object can be viewed repeatedly for several days before the scanning moves on across the sky.

\section{SINGLE STAR VS. MULTIPLE STAR}

The data processing outlined above shows no distinction with the nature of the object observed. It seems to apply in the same way to single and multiple stars. However the truth is not that simple. The HIPPARCOS photometric system is not defined by the actual instrument filter, but by a set of photometric standard stars defined before the mission and carefully monitored to this aim. The main reason which forced this choice is the fact that the HIPPARCOS photometric response is time dependent, both in sensitivity and chromaticity. Hence, one cannot rely on the true filter to build a consistent photometric scale over the three of four years of the mission. The calibration model, fitted to the observations of standard stars, allows to determine the transformation from the actual filter to the conventional one. As this transformation involves the use of the color, the final results depends slightly on the knowledge of the star color. While this may be adequately known for single stars, it is generally less reliable for multiple stars, and in addition poorly defined when the components are of different spectral types. For this reason the magnitude obtained in the case of a multiple star, observed as a single object, is not as accurate as for a single star. Obviously the degradation is a function of the magnitude difference and the separation projected on the grid of HIPPARCOS. Table 2 lists the precision within each class of magnitude, irrespective of the other double star characteristics. The numbers quoted refer to the magnitude of a multiple system as a whole and not to its components taken separately and to an average of 25 passages of the star in one of the HIPPARCOS field of view.

TABLE 2. Photometric precision for multiple systems after one year of mission in $10^{-3}$ mag. The magnitude refers to the unresolved object.

\begin{tabular}{|lllllllllll|}
\hline Magnitude & 4 & 5 & 6 & 7 & 8 & 9 & 10 & 11 & 12 & 13 \\
Precision (0.001 mag) & 1.8 & 1.9 & 2.2 & 2.4 & 2.8 & 4.2 & 8.5 & 11 & 14 & 40 \\
\hline
\end{tabular}

The second difference between single and multiple star signals lies in the amplitude of modulation $I M$ and $I N$ in Equation 1. The coefficients $M$ and $N$ are well known for single stars which allows to derive an intensity estimate:

$$
I_{a}=\frac{(I M) m+(I N) n}{m^{2}+n^{2}}
$$




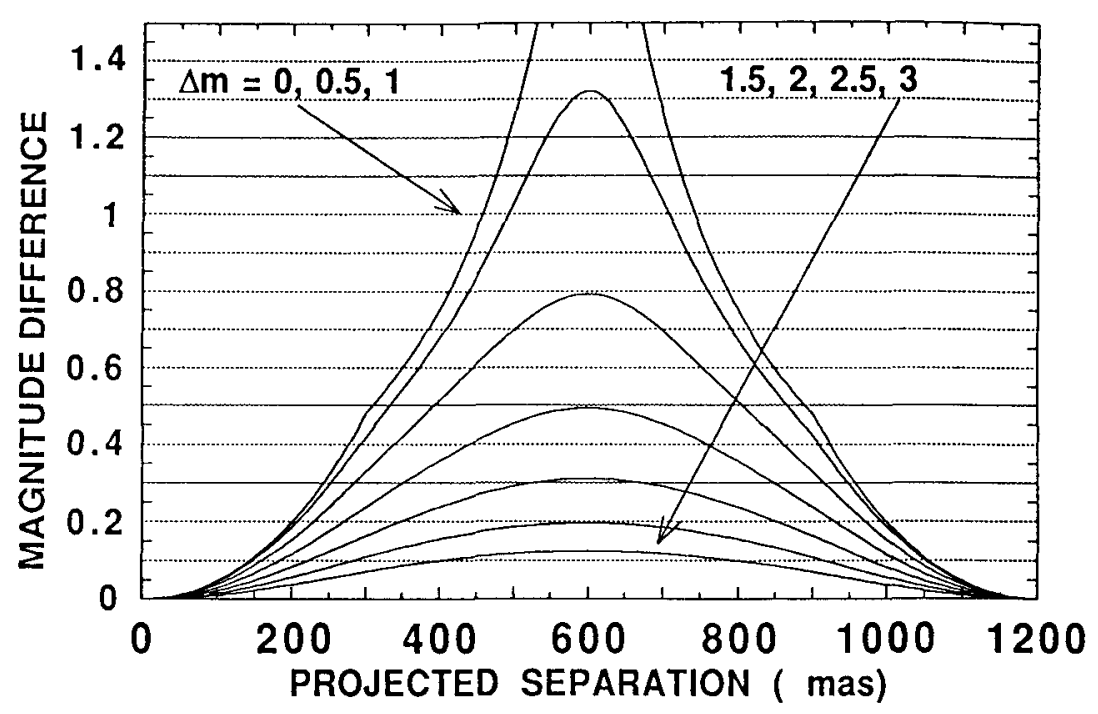

FIGURE 1. Ratio of the intensity estimates $I$ and $I_{a}$ expressed in magnitude as a function of the separation projected on the grid, in 0.001 arcsecond. $\Delta m$ refers to the magnitude difference between the components.

where lower case letters denote estimates of the corresponding true values for the single star model.

The modulation factors $M$ and $N$ are highly dependent on the intensity distribution on the star image, and thus of the structure of the object down to $0^{\prime \prime} .1$. For example, Figure 1 shows the variation of the intensity estimate $I_{a}$ referred to the single star value as a function of a double star parameter. The deviation from the single star model starts smoothly at small separations and becomes significant for projected separations larger than 100 mas. Clearly this deviation prevents from using the photometric information contained in the amplitudes of modulation to determine the star magnitude.

Actually, we do take advantage of this effect to recognize unknown double stars by comparing the intensity $I_{a}$ computed with the amplitudes $I M$ and $I N$ to the intensity derived from the average photon counts $I+B$, after removal of an estimate of the background. The difference expressed in magnitude is shown in Figure 1, and serves as a basis of a powerful recognition test. An application of this test to a subset of stars known to be double is illustrated in Figure 2.

For noiseless data of a single star, all data points would fall on the line $\Delta m=0$. Typical scatter due to the noise is less than $0.05 \mathrm{mag}$. All observations which give positive $\Delta m$ larger than the natural scatter are due to the star not being single. It is clear that many double stars are recognized by this test. 


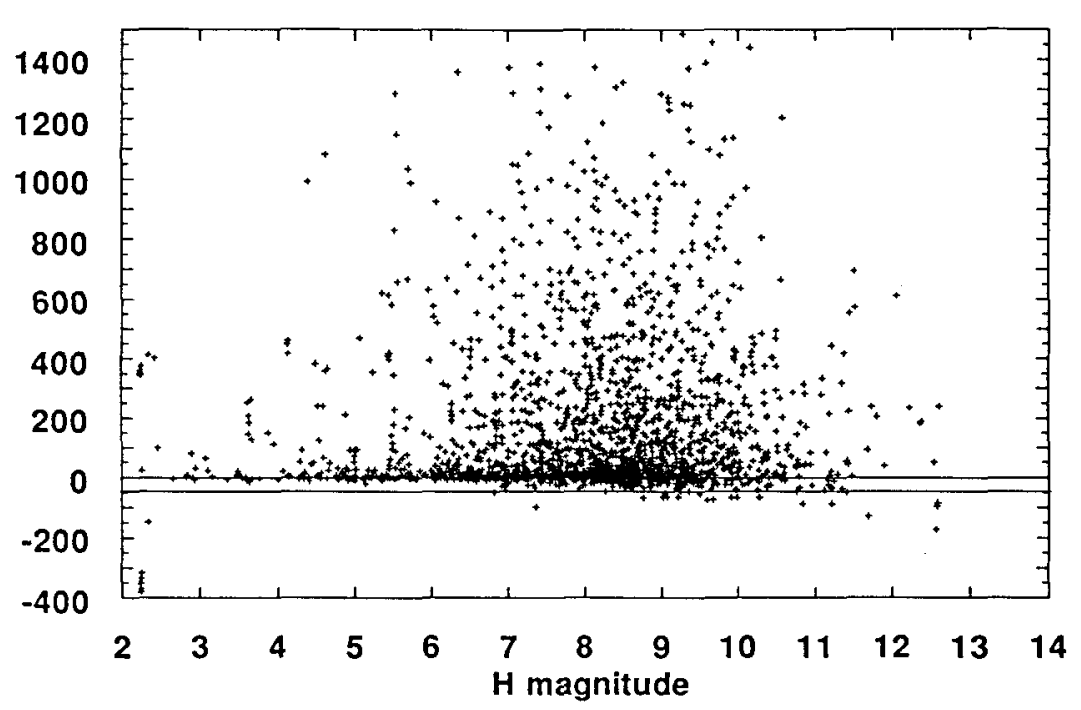

FIGURE 2. Recognition of non-single stars with the photometric test. Single stars appear in the vicinity of the bottom line. All dots scaterred with a positive magnitude difference (in $0.001 \mathrm{mag}$ ) indicate that a mutltiple star is detected.

\section{PHOTOMETRIC SOLUTION FOR THE COMPONENTS}

We take up now the much more involved problem of resolving the signal of a multiple system into that of its components. The discussion is restricted to the photometric aspect, the astrometric solution being treated in a companion paper.

As a result of the linearity of the HIPPARCOS detector, when two or more star images are simultaneously on the sensitive part of the detector, their contributions simply add. In the case of a double star we can write the signal of the primary and secondary as, (disregarding the background noise)

$$
\text { and } \quad \begin{aligned}
S_{1}(t) & =I_{1}+I_{1} M_{1} \cos \left(\omega t+\phi_{1}\right)+I_{1} N_{1} \cos \left(2 \omega t+\psi_{1}\right) \\
S_{2}(t) & =I_{2}+I_{2} M_{2} \cos \left(\omega t+\phi_{2}\right)+I_{2} N_{2} \cos \left(2 \omega t+\psi_{2}\right) .
\end{aligned}
$$

With the assumption that the two components are single stars, we have the two additional constraints on the phases, $\psi_{1}=2 \phi_{1}, \psi_{2}=2 \phi_{2}$, after instrumental corrections are applied. The phase difference $\alpha=\phi_{2}-\phi_{1}$ denotes the projected separation on the grid between the primary and the secondary, modulo one gridstep ( 1 gridstep $=1^{\prime \prime}: 208$ ).

Then, the global signal for a double reads,

$$
S(t)=S_{1}(t)+S_{2}(t)
$$

and takes the form given in Equation 1 with the following correspondence readily obtained with complex notation, 


$$
\begin{aligned}
I & =I_{1}+I_{2} \\
I M \cos \phi & =I_{1} M_{1} \cos \phi_{1}+I_{2} M_{2} \cos \phi_{2} \\
I M \sin \phi & =I_{1} M_{1} \sin \phi_{1}+I_{2} M_{2} \sin \phi_{2} \\
I N \cos \psi & =I_{1} N_{1} \cos 2 \phi_{1}+I_{2} N_{2} \cos 2 \phi_{2} \\
I N \sin \psi & =I_{1} N_{1} \sin 2 \phi_{1}+I_{2} N_{2} \sin 2 \phi_{2} .
\end{aligned}
$$

The summation in the right-hand side would extend over the number of components for a multiple system with more than two stars. Briefly, the lefthand side is known at each observation while the parameters in the right hand side refer to the two components and are unknown. It is in principle possible to solve this system for the unknowns $I_{1}, I_{2}, \phi_{1}, \phi_{2}$ at each star transit. But the non-linearity of the system yields very bad statistical properties of the solution with bias which cannot be corrected easily and increases with the variance of the measurements. The only way out is to postpone the resolution of the nonlinear system when enough data have been acquired. One may see that the phase difference $\phi_{2}-\phi_{1}$, that is to say the projected separation reduced to one gridstep, varies slowly with the scan direction, all the more as the double star separation is small. It is therefore possible to accumulate left-hand sides to improve the statistical reliability and solve for the intensity ratio $I_{2} / I_{1}$ and the phase difference $\phi_{2}-\phi_{1}$. The latter is to be used for the astrometric solution, while the former leads to the magnitude difference with

$$
\Delta m=-2.5 \log \frac{I_{2}}{I_{1}} .
$$

The trade off between the number of transits used in the accumulation to decrease the variance and the additional scatter resulting from the varying geometrical configuration between the grid and the binary star is one of the most sensitive parts of the software and depends on the a priori knowledge of the separation. To illustrate this point, consider an average situation. From one observation to the following two hours later, the scanning direction is inclined to the previous by about $1^{\circ}$. Hence for a double star of separation on the sky of $5^{\prime \prime}$, the difference between the two projected separations may be as large as $5 \times \sin \left(1^{\circ}\right) \simeq 0^{\prime \prime} .09$, much larger than the variations induced by the noise. Therefore for these stars with large separation, the accumulation has to be limited to the two observations obtained $20 \mathrm{~min}$ apart of each other. On the other hand for a close binary one can accumulate over two or three consecutive scans and thus decreases, the magnitude of the statistical noise before solving the non-linear system and limit in this way the risk of bias.

The solution of Equations 8-11 ends up with the projected phase difference $\phi_{2}-\phi_{1}$ for the particular scanning direction and the ratio $I_{2} M_{2} / I_{1} M_{1} \simeq I_{2} / I_{1}$. The former will be an input to the astrometric solution, while the latter is the same as the magnitude difference between the two components. Eventually, with the magnitude difference and the magnitude of the system as a whole one computes the magnitude of each component together with their standard deviations. At a later stage, these magnitudes obtained on different scan circles are collected together into a single accurate magnitude for each component, provided they are not variable stars. 


\section{RESULTS AND DISCUSSION}

We have processed along the lines given in the preceding section, about one year of data. This amounts to about $3 \times 10^{6}$ field transits for 120,000 stars. Among these stars, a subset of 16,000 have been detected as non-single and diverted to the double and multiple star processing. On the average, a star was observed over 25 field-transits, but on only 5 to 7 distinct scanning directions. A photometric solution has been found for all these stars, both as non-resolved objects and when consideed as a binary star. In this case a magnitude for each component is searched for.

Results as a function of the magnitude of the considered component are summarized in Table 3 . They must be looked at with reference to T-able 2 which gives the same results for the unresolved object. The loss due to the resolution into components appears clearly, and may be estimated to a factor 3 for the primary an to 7 for the secondary. In fact in the latter case the scatter is quite important and depends on the magnitude difference. Comparison with stars with statistically reliable ground-based photometry indicates that our photometric solution is not reliable for magnitude difference larger than $3 \mathrm{mag}$.

TABLE 3. Photometric precision for primary and secondary stars in $10^{-3} \mathrm{mag}$. The magnitudes refer either to that of the primary or secondary.

\begin{tabular}{|llllccclll|}
\hline Magnitude & 4 & 5 & 6 & 7 & 8 & 9 & 10 & 11 & 12 \\
Primary (0.001 mag) & 2.2 & 4.1 & 4.3 & 6.0 & 7.8 & 12.4 & 19.0 & 22 & 26 \\
Secondary (0.001 mag) & 3.5 & 6 & 9 & 15 & 15 & 25 & 40 & 60 & 90 \\
\hline
\end{tabular}

A similar analysis was carried out for the magnitude of the components as a function of the magnitude difference, for a subset of stars with global magnitude in the range 7.5 to 9 . The results are illustrated in the plots of Figure 3. As expected the larger the magnitude difference between primary and secondary, the less accurate the solution of the secondary. On the other hand the quality of the global photometry is nearly recovered for the primary. For components of comparable brightness, the loss in precision is significant, going from $0.004 \mathrm{mag}$ for the unresolved system to $0.02 \mathrm{mag}$ for either component.

\section{CONCLUSION}

The signal recorded during the transit of a star on the grid of HIPPARCOS contains a wealth of information related to the astrometric and photometric parameters of double stars. We have devised specific algorithms to extract that information and calculate the HIPPARCOS magnitude of each component. This has been successfully achieved for 16,000 stars observed during the first year of mission and recognized as non-single. The accuracy for an unresolved object of $8^{\text {th }}$ mag is typically $0.003 \mathrm{mag}$, but degrades significantly when resolved into its components. 


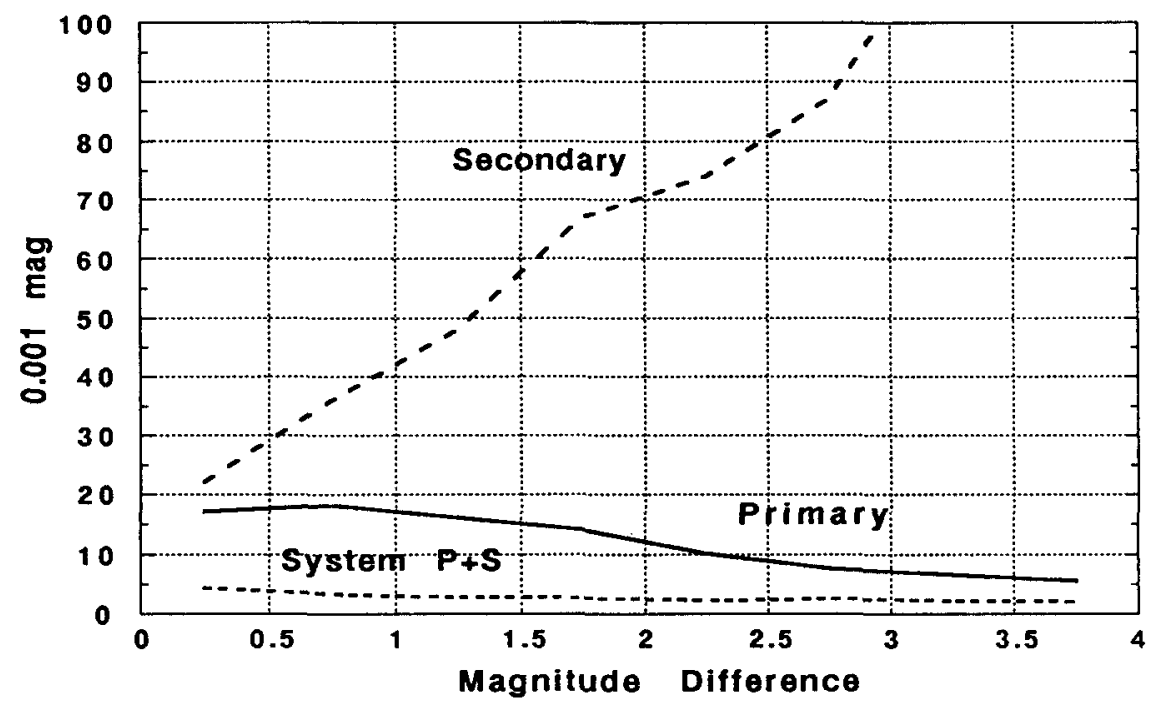

FIGURE 3. Mean of standard deviation in the magnitude of the primary, secondary and whole system as a function of the magnitude difference.

\section{REFERENCES}

Grenon, M., Mermilliod, M., \& Mermilliod, J.C. 1991, "The HIPPARCOS Input Catalogue III. Photometryn, $A \& A$, to appear May 92

Mignard, F., Borriello, L., Kovalevsky, J., Prezioso, N., \& Bernacca, P.L., 1989, "Double Star Reduction in FAST ${ }^{n}$, in The HIPPARCOS Mission, ESA-SP 1111, III, 243

Mignard, F., Froeschle, M., Falin, J.L. 1986, "Photometry with IDT II: Double stars", in Proc. of the Bari coll. Processing of Scientific Data from HIPPARCOS, Third FAST Thinkshop, eds. P.L. Bernacca and J. Kovalevsky, p. 263

Mignard, F., Froeschlé, M., Badiali, M., Cardini, D., Emanuelle, A., Falin, J. L., \& Kovalevsky, J. 1992, "HIPPARCOS double star recognition and processing", $A \& A$, to appear May 92

Murray et al. 1989, "Analysis of the Image Dissector Tube Data", in The HIPPARCOS Mission, ESA-SP 1111, III, 33 